\title{
The Strategy of Advanced Analysis in Semiconductor Nano-Device: From Nanoprobing to Nanoscopy and Nanoanalysis
}

\section{LiLung Lai}

Semiconductor Manufacturing International (Shanghai) Corp.18 Zhangjiang Road, Pudong New Area, Shanghai, PRC

As semiconductor devices shrink down to $20 \mathrm{~nm}$ and below, the required imaging and analysis become more critical and challenging. To support the analytical purposes, the Nanoprobing (Figure 1) from SEM/AFM system provides the capability of direct measurement for Nano-device. We postulate the uniqueness [1] for the link in between electrical Nanoprobing and physical Nanoscopy \& Nanoanalysis. Regarding the Nanoscopy and Nanoanalysis, we need capabilities for the dopant profiling and inspection for new materials, HKMG, and new structure, 3D FinFET. There are already plenty of references for the progress $[2,3]$. In this paper, I would emphasize on the power of Atom Probing Tomography (APT), Analytical TEM (EELS and XEDS) and Atomic resolution STEM for the further physical analysis of post-Nanoprobing. Figure 2 illustrates the capable solutions of Nanoscopy and Nanoanalysis to reveal doping profile and gate materials in the MOS device. Therefore, the progress of Nanoscopy and Nanoanalysis becomes valuable for the advanced Semiconductor analysis.

The latest SDD-EDS with high-throughput of X-ray signal is able to detect Arsenic (As) at the limitation of $0.02 \%\left(\sim 1 \times 10^{19} / \mathrm{cm}^{3}\right)$ and upper to around $0.1 \%\left(\sim 5 \times 10^{19} / \mathrm{cm}^{3}\right)$ for Phosphorus (P) in N-type Silicon [4]. Figure 3 shows the strategy of dopant profiling with compensation of XEDS_in_STEM \& SCM. However, it is inactive for Boron (B) in the P-type Silicon, even we use windowless detector (Figure 2). EELS can do it but not easy for the analysis [5]. Because the atomic-level resolution of STEM Nanoscopy and X-ray Nanoanalysis, it can reveal the small dimension of high dose (N+/P+/LDD) area, the development of low-kV performance, big collection angle and high take-off angle X-ray detection could be strategic investment.

Atom Probing Tomography (APT) has become emerging technique to show 3D characterization of atomic distribution for different elements and doping within the very small needle by the proper FIB preparation [6]. The sensitivity for the doping is about order of $10^{18} / \mathrm{cm}^{3}$ and detectable for channel doping in very small transistors [7]. It will become an inevitable technique for smaller device analysis and $3 \mathrm{D}$ transistor. However, the specimen preparation is very challenging and difficulty for the site-specific analysis. The more applications are under in progress.

The detection limit of APT would be several tens of atoms in the analytical region. Therefore, we will face the next challenging of getting smaller device at post-10nm era. The directly atomic imaging by high resolution HAADF-STEM or other TEM modes should become next generation of Nanoscopy.

No matter of Nanoscopy (TEM/STEM) and Nanoanalysis (EDS/EELS/APT) for semiconductor advancing technology, the Focused Ion Beams (FIB) is inevitable tool for the specimen preparation [8]. Thus, the developing of high resolution FIB, especially for Low-kV performance, should become urgent topic for the demand of semiconductor applications. 


\section{References:}

[1] LL Lai, International Symposium of Testing and Failure Analysis 2013, p.222

[2] "Transmission Electron Microscopy in Micro-nanoelectronics", Ed. Alain Claveri, (ISTE) (2012)

[3] F. Panciera et al., Applied Physics Letter 100 (2012), 201909

[4] R Pantel, Ultramicroscopy 111 (2011), p.1607

[5] M. Duchamp et al., Journal of Applied Physics 113 (2013), 093513

[6] A.K. Kambhama et al., Ultramicroscopy 111 (2011), p.535

[7] H. Takamizawa et al., Applied Physics Letter 100 (2012), 25350

[8] K. Thompson et al., Ultramicroscopy 107 (2007), p. 131

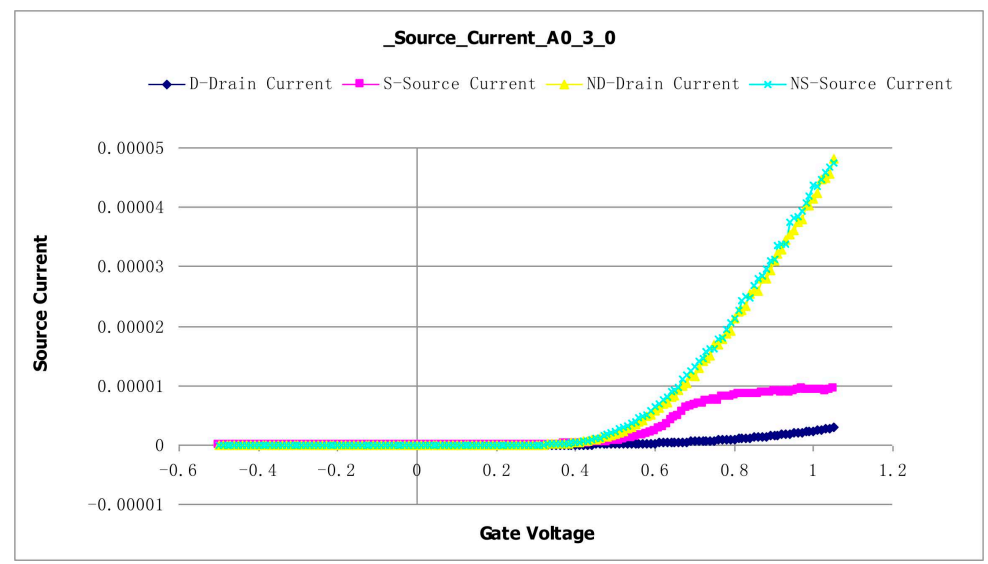

Figure 1. Example of Nanoprobing data for defect transistor

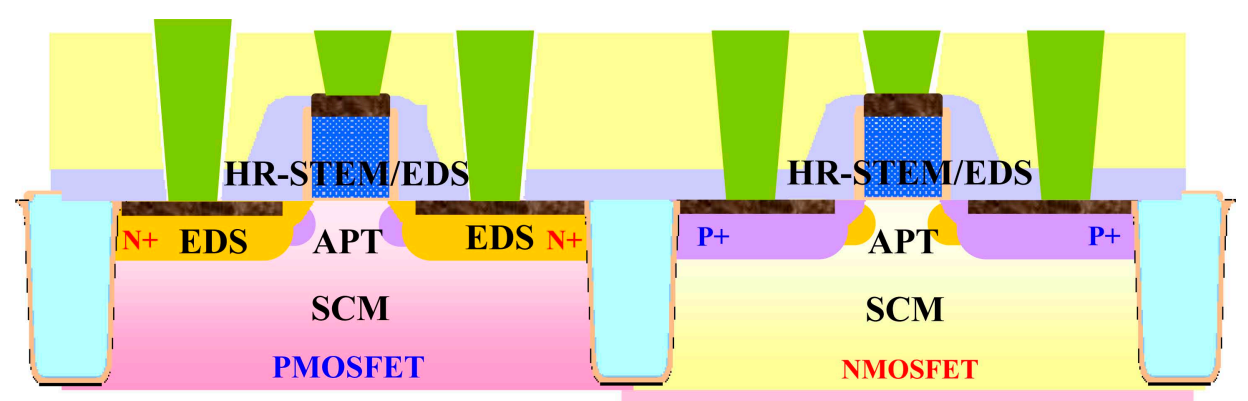

Figure 2. Different Nanoscopy and Nanoanalysis is suitable for different material and doping area. Note: XEDS/EELS in STEM are almost incapable for Boron detection.

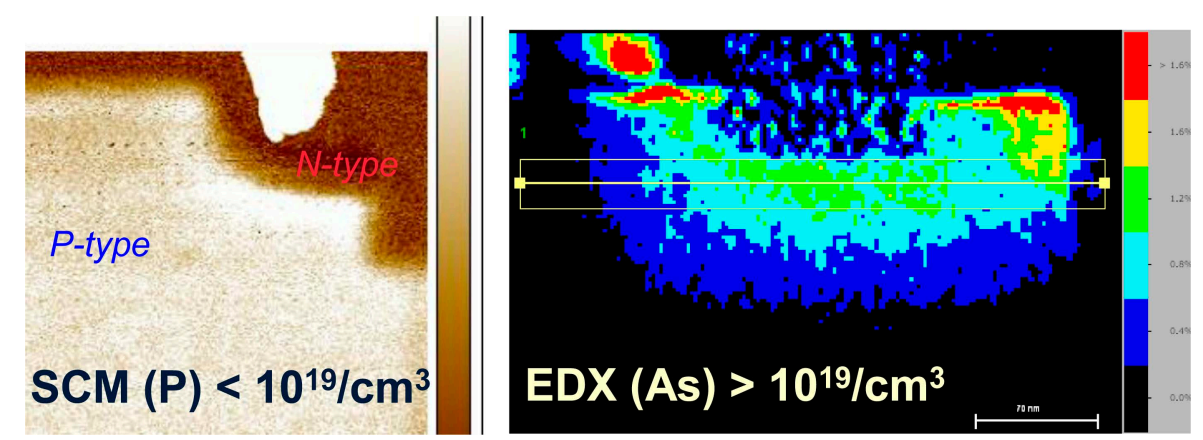

Figure 3. Using (SDD)XEDS-in-STEM for dense doping and SCM for rare doping 